Sharif University of Technology
Scientia Iranica
SCIENTIA
I RAN I C A
http://scientiairanica.sharif.edu

\title{
Indirect structure damage identification with the information of the vertical and rotational mode shapes
}

\author{
M. Ramezani and O. Bahar* \\ International Institute of Earthquake Engineering and Seismology (IIEES), Tehran, P.O. Box 19395-3913, Iran.
}

Received 21 September 2020; received in revised form 7 December 2020; accepted 8 May 2021

\author{
KEYWORDS \\ Indirect damage \\ identification; \\ Rotational mode \\ shapes; \\ Improved genetic \\ algorithm; \\ Model updating; \\ Modal strain energy.
}

\begin{abstract}
The present study aims to propose a robust method to detect the damage severity and location of the structural elements, focusing on the data type and acquisition method and promoting the model updating tools. The novelty of this method lies in its rotational mode shape acquisition that provides valuable information on the damage. In this method, the damaged elements were indirectly identified by detecting the healthy elements and eliminating them from the search space. Moreover, this method could minimize the modal strain energy difference between the damaged reference model and the numerical model using an optimization algorithm. An improved genetic algorithm was then employed to perform the optimization task. In this study, four numerical and two experimental damage scenarios were applied to a simply supported beam to examine the performance of the proposed method. Data acquisition systems were implemented using vision-based and accelerometer-based methods. The results indicated that this method could accurately identify the location and severity of damage using only the first mode shape since the rotational mode shapes were more sensitive to damage than the vertical mode shapes.

(C) 2021 Sharif University of Technology. All rights reserved.
\end{abstract}

\section{Introduction}

Significant damages to civil infrastructures caused by recent natural disasters [1,2] have highlighted the significance of timely damage identification in structural systems. Any undesirable change in the material properties or geometry of the structure is regarded as a damage. The age of structures, element deterioration, natural disasters, wrong use, and lack of proper maintenance are the main causes of damages. In case the damages are not detected, the structure may collapse. Visual inspections and traditional non-destructive tests fail to detect the damages hidden in the nonstructural elements. Therefore, a global method is required to detect damages at any inaccessible location. Structural

\footnotetext{
*. Corresponding author. Tel.: +982122831116
} E-mail address: omidbahar@iiees.ac.ir (O. Bahar) damages reduce the stiffness of one or more elements. The damage in elements would change the structural dynamic properties such as the modal frequencies and mode shapes. The dependency of the dynamic properties of structures (i.e., natural frequencies and mode shapes) on the physical properties (i.e., mass and stiffness) has led to development of some methods for health monitoring of the structures. Contaminated measured data and low sensitivity to damages have hindered the development of basic/primitive methods [3]. Use of convenient tools for measuring the data or the presence of significant damages can enhance the performance of these methods [4]. With regard to the limited performance of the basic/primitive methods, researchers have developed methods such as mode shape curvature, Modal Strain Energy (MSE), model updating, flexibility-based methods, etc. that could provide more accurate information about the location [5-9].

A number of methods that use output-only data 
for damage detection require much information and a large number of vibration modes, and their performances may be significantly attenuated in case of any reduction in the amount of available information [10]. Based on what was mentioned, Pandey and Biswas [7] determined the location of damage using a limited number of modes. Yang and Liu [11] proposed an algorithm based on a change in the flexibility of matrices that could determine the location and severity of damages. Li et al. [12] reduced the effect of highermode elimination by adding the generalized flexibility matrix instead of the global flexibility matrix.

In recent years, MSE has been the main focus of a number of studies on the structural health monitoring. This parameter, composed of the element stiffness and mode shapes of structures, is highly sensitive to the presence of damage in the structure. Shi et al. [13] proposed a method based on MSE variations in damaged and healthy elements. In their proposed method, elements with a significant change in MSE were regarded as potentially damaged elements. The location and severity of damages were also determined through a quantifying step. Later, Moradipour et al. [14] improved this method by reducing numerical errors.

Homaei et al. [15] proposed a direct damage detection method based on the criterion of mode shapes. Their method enjoyed several advantages namely simplicity, applicability to a large number of elements, accuracy of the location of damage, sensitivity to low damage severity, estimation of the number of required mode shapes, and low sensitivity to noise-contaminated data. They investigated the viability of their proposed method using three beam examples over different time spans.

Yan and Ren [16] proposed a method based on the sensitivity of the modal flexibility that used multiple modes for damage detection. The merit of their method was its ability to eliminate the adverse effect of truncating higher-order modes. Their proposed method was evaluated using two beams in the presence of noise in mode shapes. Yazdanpanah and Seyedpoor [17] introduced a new damage indicator based on the mode shape data. The mode shapes as well as their slope and curvature before and after damage were taken into account in damage detection. Their method was verified by two beams and multiple mode data. Shahri and Ghorbani-Tanha [18] considered the ratio of modal kinetic energy change in their study with low sensitivity to common noise. Their method was verified using a beam-like structure considering several required modes and a sensitivity matrix was calculated by the closedform sensitivity of eigenvalues of the structure.

Many metaheuristic algorithms developed to solve optimization problems can be used for damage detection such as Genetic Algorithm (GA) [19], Par- ticle Swarm Optimization (PSO) [20], Artificial Bee Colony (ABC) algorithm [21], tug of war optimization algorithm [22], Differential Evolution (DE) algorithm [23], Enhanced Vibrating Particles System (EVPS) algorithm [24], and Water Evaporation Optimization (WEO) algorithm [25], etc. These algorithms can search the space for possible answers without solving equations. The convergence speed, finding global optimum, accuracy of answers, and type of objective function determine the most appropriate tool for damage detection. Gomez and Silvia [19] investigated two damage detection methods including a frequency sensitivity-based method and GA. They compared these methods and achieved similar results. Some first frequencies were required in their method to evaluate the damage location and severity.

Nobahari and Seyedpoor [26] proposed a GA modified by two new operators which could increase the speed of convergence. The objective function of their proposed algorithm was based on correlation which required several first frequencies. Seyedpoor [20] proposed a two-stage method to identify the location and severity of damages. At the first stage, the location of damaged elements was identified by the MSE-based index. Then, the severity of damages was determined by PSO at the second stage. Xu et al. [21] presented a method based on a chaotic ABC. Their proposed method had some advantages such as ease of implementation and good robustness. To overcome the shortcoming of this method, i.e., the slow convergence rate, they utilized the tournament selection mechanism instead of the roulette mechanism. Furthermore, they introduced a chaotic search mechanism. The objective function of this method used the residuals of natural frequencies and modal assurance criteria, which required multiple-mode data. Seyedpoor et al. [23] used an improved DE algorithm as a tool for detecting location and severity of damage. The objective function of their method was defined in terms of changes in natural frequency. Their proposed model was verified by an experimental model and two numerical examples with the consideration of measurement noise. Toloue et al. [27] used the damage load vectors computed by variations in intact and faulty flexibility matrices for damage detection of a frame. In this method, acceleration responses were taken into account to derive the flexibility matrix.

Kaveh et al. [24] employed the EVPS optimization algorithm to detect damages in truss structures and compared the results of this method with those obtained from the vibrating particles system algorithm. According to this algorithm, each response is assumed to be a single-degree-of-freedom system with viscous damping approaching its equilibrium point. The authors employed the modal shape and frequency information to define the objective function 
and evaluated this method for four truss structures based on the information of the first few modes. In order to reduce the computational efforts during the optimization procedure, Kaveh et al. [25] proposed a two-phased method. In the first phase, the structural frequencies are calculated; in case the frequencies are equal, the algorithm moves on to the second phase and determines the modal shapes. As a result, the number of cases under study would decrease. They applied this idea to the accelerated WEO algorithm and identified the damages to the beam, frame, and three-dimensional structures.

To establish an accurate finite element model, Liang et al. [28] proposed a model updating method by combining the cross-model and cross-mode. They employed natural frequencies to update the model owing to their ease of measurement, high precision, and insensitivity to noise. They verified the proposed algorithm using a numerical model and two actual shear structures. Khatir et al. [29] proposed a twostage approach to damage detection. At the first stage, the potential damaged elements were identified by the local frequency change ratio indicator. To detect damage severities, the teaching-learning-based optimization algorithm was utilized at the second stage. They compared their method with PSO and bat algorithm. Mishra et al. [30] used an ant lion algorithm, a population-based search algorithm, with fewer parameters than other meta-heuristic algorithms for structural damage detection. The objective function of their method used multiple vibration data such as some first mode shapes and natural frequencies. Krishnanunni et al. [31] studied how natural frequencies and static displacements could change in case of the occurrence of the structural damage. They employed the cuckoo search algorithm that used vibration data and static displacement measurements for damage detection. Their method was evaluated using cantilever and fixed both ends of the beams, which required some mode data to meet its objective function.

Inclinometers, or rotation sensors, which are widely used in industries such as automotive, aerospace, and electronics, are designed to measure angular rotation. These sensors are designed based on the pendulum behavior caused by gravity. The pendulums used in inclinometers are categorized into three types, i.e., solid masses, liquids, and gases, which can be measured in a variety of ways. Over the past years, the performance and accuracy of inclinometers have considerably improved, making them suitable for damage detection applications. The sampling rate of the inclinometers decreases with an increase in the measurement accuracy. However, the application of the proposed method to collect rotational data is part of the novel methodology presented in this study which examines whether it can be used for damage iden- tification. Although the application of inclinometers or rotational responses to understanding the behavior of the structure has proved to be effective, limited studies have been conducted on the direct measurement of rotation to identify the damage [32-35], mainly due to the limitations of sensor technology such as lack of commercially accurate sensors and sampling limitations.

Alten et al. [32] employed acceleration data to detect the damage with emphasis on frequency changes. They also investigated the effect of damage on the rotation increase. In another study, they examined the sensitivity of the rotation to the damage in bridge through numerical and experimental analyses. They proposed a damage detection method based on the deformation area difference, which could detect damage through an area difference in the rotation diagrams of healthy and damaged structures under static loading. Huseynov et al. [35] evaluated the behavior of a simply supported beam numerically and experimentally using the direct measurement of rotation in healthy and damaged structures (difference in rotation influence lines). They employed the difference in rotation influence lines as a criterion for damage identification. In their study, the effect of rotation sensitivity to damage as well as the position of sensors to sensor sensitivity were studied. The mentioned authors used a high-grade uniaxial accelerometer to measure the rotation. They showed that the use of modal data alone for damage detection in their model was not entirely successful, and that use of strain gauge sensors could partially detect the damage in case the strain gauges were installed near the damage location. However, the results of measuring the rotation showed the damage in all studied scenarios. In a similar study, Hester et al. [34] directly measured the rotation in a simple beam. First, they examined the sensitivity of the rotation as a damage index through a numerical study. This method was then experimentally studied on a simple beam. Finally, their proposed method was evaluated on a bridge with a simply supported span of 20 meters. Acceptable results were obtained from damage assessment.

Several studies have contributed to the introduction of damage detection methods. Among them, the method that can precisely determine the damage severity of elements with a minimum scope of information is the best one. The main objective of this study is to accurately localize the damaged elements and quantify their severities using only the first vibration mode shape. Despite the benefits of rotation measurement in terms of damage identification, limited studies have been conducted on the direct measurement of this parameter. In the present study, modal information was extracted through the displacement and rotation signals acquired by the vision-based method and accelerometers. Behind this method lies some novel 
ideas such as extraction of rotational mode shapes and indirect damage detection that finally lead to the establishment of a robust damage detection method capable of accurately finding damage severities using only the first vibration mode shape without noise and obtaining acceptable results using noise-contaminated data. At the updating stage of GA, the experiences concerning the destruction of actual structures such as damage occurrence in the limited number of elements were included to increase the convergence speed and identification accuracy. In general, this method found a solution to decreasing the number of required vibration modes.

\section{Theoretical background}

Modal characteristics of the undamped linear systems are described by the following equation (the eigenvalue equations):

$$
[K]\left\{\Phi_{i}\right\}=\lambda_{i}[M]\left\{\Phi_{i}\right\}
$$

where $[K]$ and $[M]$ are the $N_{D} \times N_{D}$ global stiffness and mass matrices, respectively; $N_{D}$ indicates the number of Degrees Of Freedom (DOFs) of the system; $\lambda_{i}$ and $\left\{\Phi_{i}\right\}$ are the natural frequency and the $i$ th vibration mode shape of the system, respectively. While each node in the two-dimensional model has three DOFs including two transitional and one rotational DOFs, each node in the three-dimensional model has six DOFs including three transitional and three rotational DOFs. Mode shape or natural vibration shape indicates how the points defined in the structure or the DOFs of the structure would change when vibrating at the natural frequency of the structure. In fact, the natural frequencies and mode shapes show how a structure vibrates under dynamic loads. Although the structural response is a function of the input excitation, it is essentially composed of a set of mode shapes with different participation factors. This is the reason why extracting the mode shape of the structure is independent of the type and intensity of the excitation. In case of damage occurrence in the structures caused by overloading, fatigue, etc., the dynamic characteristics would consequently change due to their dependence on physical characteristics, as shown in Eq. (1). On the contrary, structural damages often reduce the stiffness of one or more elements throughout the system and rarely decrease the mass of the structure. Therefore, mass changes were ignored in this study.

The vibration equation of the damaged system is defined as follows:

$$
[K]^{d}\left\{\Phi_{i}^{d}\right\}=\lambda_{i}^{d}[M]\left\{\Phi_{i}^{d}\right\}
$$

where superscript " $d$ " denotes the damaged state.
The global stiffness of the system is obtained from the total elements of the system, as shown in the following:

$$
[K]=\sum_{j=1}^{N_{E}}[K]_{j},
$$

where $[K]_{j}$ is the $N_{D} \times N_{D}$ stiffness matrix of the $j$ th element in the global stiffness, and $N_{E}$ is the total number of elements used in forming global structure matrices. Therefore, the effect of damaged elements in the global stiffness matrix is defined through the following equation:

$$
[K]^{d}=[K]+\sum_{j=1}^{N_{E}} \Delta[K]_{j}=[K]+\sum_{j=1}^{N_{E}} \alpha_{j}[K]_{j},
$$

where $\Delta[K]_{j}$ is the difference in the stiffness in both healthy and damaged states and $\alpha_{j}$ is the stiffness reduction coefficient of the $j$ th element. Since the element stiffness in the damage state is lower than the healthy state, the stiffness reduction coefficient is considered as $-1<\alpha_{j} \leq 0$.

\subsection{Calculating MSE of elements}

MSE, measured by mode shapes and stiffness, is an entirely sensible parameter for damage detection. The MSE values for the $i$ th mode of vibration and the $j$ th element in healthy and damaged states are defined through the following equations [9]:

$$
\begin{aligned}
M S E_{i j} & =\frac{1}{2}\left\{\Phi_{i}\right\}^{T}[K]_{j}\left\{\Phi_{i}\right\}, \\
M S E_{i j}^{d} & =\frac{1}{2}\left\{\Phi_{i}^{d}\right\}^{T}[K]_{j}^{d}\left\{\Phi_{i}^{d}\right\} .
\end{aligned}
$$

Due to the unknown damage severity at first, the stiffness estimated by the proposed algorithm is considered for calculating the MSE of the damaged state.

The MSE of the entire structure obtained from the MSE of the whole elements for the $i$ th mode of vibration is defined as follows [20]:

$$
M S E_{i}=\sum_{j=1}^{N_{E}} M S E_{i j}
$$

\section{Optimization algorithm}

The GA that contains some processes such as reproduction, crossover, and mutation is nature driven. This algorithm is such a powerful optimization tool that searches the problem space randomly and purposefully. After every step, GA finds even better answers. In case the populations obtained from the reproduction process produce inappropriate answers related to the previous 
step, the former populations will be used for the next step. Therefore, this algorithm always moves towards the target [36]. One of the most significant advantages of the GA is the parallel search, which empowers it to solve huge and nonlinear problems even if the problems have a local optimum. Therefore, for damage detection problems that comprise numerous variables with nonlinear behavior, the GA is an appropriate choice. Although probabilistic selection rules are used in the GA, the possibility of importing probable answers still exists, thus increasing the convergence speed.

\subsection{Determination of damage severity by Improved Genetic Algorithm (IGA)}

The IGA was used for damage detection purposes in this study. Since the stiffness reduction coefficient of elements has already been introduced already, $\alpha_{j}$ is defined as an output parameter of IGA. Upon achieving the $\alpha_{j}$ coefficients, the damaged elastic modulus of elements is determined through the following equation:

$$
E_{j}^{d}=\left(1+\alpha_{j}\right) E_{j},
$$

where $E_{j}^{d}$ and $E_{j}$ are the elastic moduli of the $j$ th element in the healthy and damaged states. In order to improve GA, it must be coded using MATLAB or other programming software. Based on the type of coding in this algorithm and the range of variables presented in Table 1, the chromosomes are generated by 0 and 1 . The number of chromosomes is equal to the number of population in each generation. Each chromosome contains the elastic modulus information of all elements, which should be transferred to base 10 to obtain real values that are the same as the stiffness reduction coefficients. These coefficients are converted to the elastic modulus of the updated model through Eq. (8). The obtained elastic modulus is assigned to the elements to calculate the stiffness of elements. It is necessary to calculate the MSE of the updated model through Eq. (9) in order to achieve the objective function.

$$
M S E_{i j}^{p d}=\frac{1}{2}\left\{\Phi_{i}^{p d}\right\}^{T}[K]_{j}\left\{\Phi_{i}^{p d}\right\}
$$

where superscript " $p d$ " denotes the potential damage. After calculating the MSE of the updated model via IGA, MSE changes of the $j$ th element in each mode (any desired mode) are calculated using the following equation:

$$
\Delta M S E_{i j}=M S E_{i j}^{d}-M S E_{i j}^{p d},
$$

where subscript $i$ is the number of desired modes.

In case the stiffness reduction coefficients are accurately estimated by the IGA, the value of MSE difference will be zero. Therefore, the objective of the IGA is to minimize the sum of MSE changes of all elements in any desired mode. Since the first mode of structures can usually be obtained more easily than others, this method employed it at all steps. The chromosomes that reduce the objective function are ranked as the more compatible ones with a greater chance for reproduction. The mutation operator changes binary numbers; hence, the IGA is not trapped in a local optimum.

After entering the best chromosome of the last generation to those obtained from reproduction and mutation steps based on the elitism concept, the newgeneration chromosomes are used for fitness determination.

According to Figure 1, GA is the optimization algorithm that minimizes the defined objective function to estimate the damage coefficients. However, in order to reduce the optimization time and increase the accuracy of results, several changes have been made in the usual process of this algorithm; this is the reason why this algorithm is called IGA. These changes are as follows:

1. Although, in reality, the exact number of damaged elements in structures is unknown, it can be claimed based on the experience that the number of damaged elements is smaller than the total number of structural elements. This assumption can significantly improve the performance of optimization algorithms in damage identification problems. Furthermore, since identification of the damage coefficients of structural elements begins

Table 1. The Improved Genetic Algorithm (IGA) parameters.

\begin{tabular}{ll}
\hline The population of generations & 10 \\
Maximum number of generations & 100 \\
The number of variables & 15 for Ex 1 \\
& 16 for Ex 2,4 for Ex 3 \\
The range of variables & {$[-1,0]$} \\
Mutation rate & 0.02 \\
Coding & Binary \\
Selection & Roulette wheel \\
Crossover & Uniform crossover binary \\
\hline
\end{tabular}




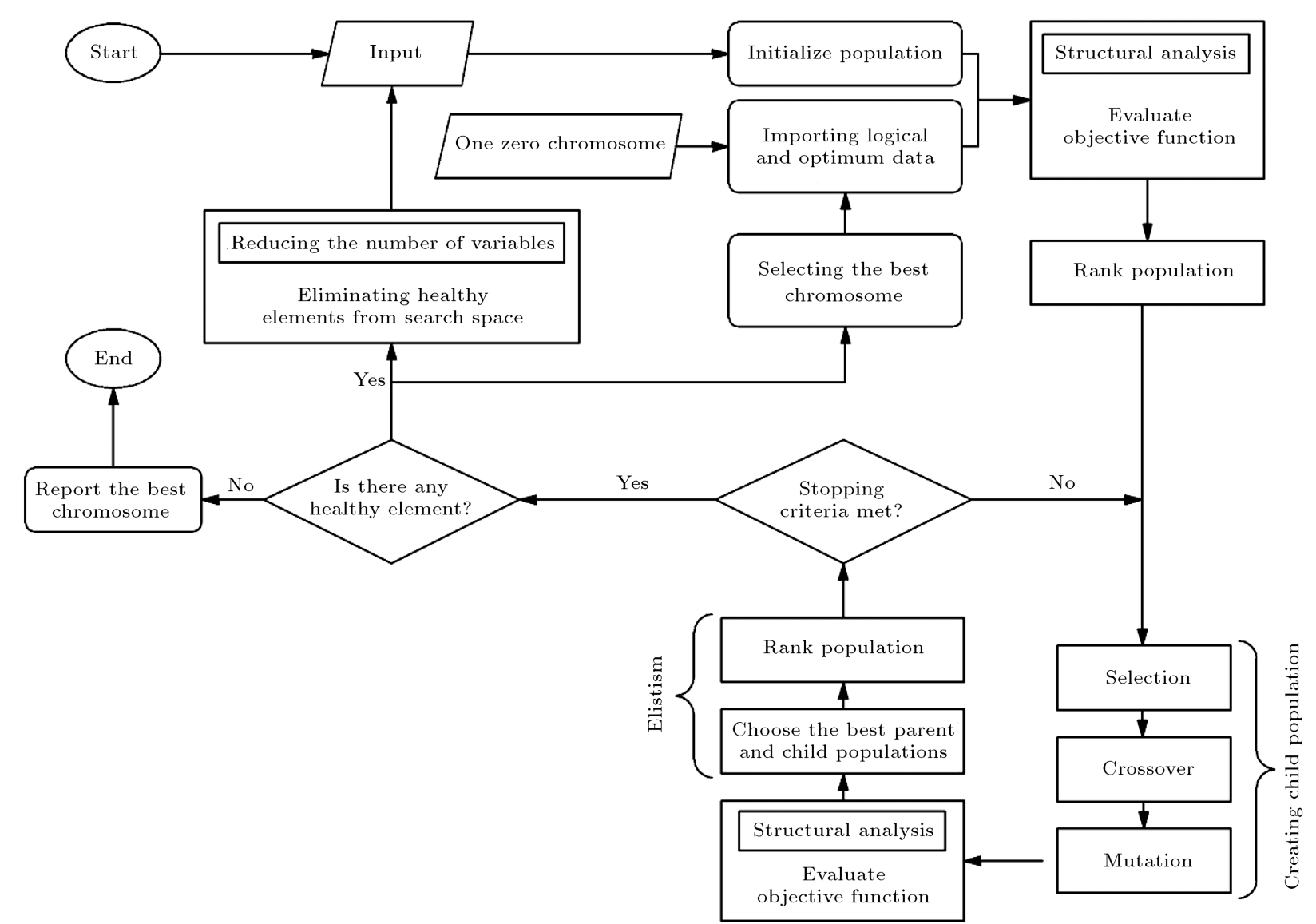

Figure 1. Determining the severity of damages in the structural elements using Improved Genetic Algorithm (IGA).

randomly, it is likely that the estimated damage coefficients at the first step are very similar to/very different from the actual values. This can affect the time required for achieving the accurate damage coefficients. Based on these remarks, one of the chromosomes at the first step is created when all the damage coefficients are equal to zero. In other words, one of the members of the produced population creates a structure whose elements are all healthy. With this change, healthy elements are identified quickly and the affected elements in the search process become the focus;

2. One of the challenges of optimization algorithms is the large number of variables. With the changes made at the previous step, the algorithm detects some of the healthy elements (according to experiences, not only the healthy elements but also the elements with no damaged element in their vicinity are quickly identified as healthy elements). By identifying these elements with no improvement made to the results in a few steps, the program removes healthy elements from the search space;

3. To start and resume the search task, one of the chromosomes that enters the initial population is chosen as the best chromosome identified from the previous search process with a larger number of variables;

4. In this algorithm, the threshold for determining healthy and damaged elements is determined as $1 \%$. This issue can be discussed from two different perspectives: (I) damage detection and (II) improvement of the optimization algorithm performance. According to the first view, all damage detection methods and techniques have false alarms, which can have different values depending on the accuracy of the method. False alarm or false positive refers to identifying a healthy element as a damaged element. This occurs for the elements that are in the vicinity of damaged elements. The ideal objective of damage detection techniques is to detect the location and severity of damages at the early stages of its formation. However, the already available damage detection methods have not achieved this objective yet due to some limitations like noises, measurement issues, sensor limitations, and physical behavior of the structure depending on different parameters. In this method, this error is kept at $1 \%$ in the analytical model. This view inspires the second view. From this point of view, the identified damages below $1 \%$ 
are not reliable. In fact, this value can be called the response reliability limit. Therefore, instead of applying the optimization algorithm to these elements, they are identified as healthy and thus, are omitted from the search process.

This process is repeated until the objective function is reduced to a specific value or a certain number of iterations. After removing the healthy elements, the convergence speed and detection accuracy increase in the next iteration of IGA as a result of reducing IGA variables. In fact, following the convergence of the IGA and removal of the healthy elements, the IGA searches the new search space in the next iteration. These iterations continue until the objective function reaches zero. The characteristics and parameter values of the IGA are presented in Table 1.

\section{Numerical simulations}

A numerical simulation was employed in this study to evaluate the performance of the proposed method. This example includes a simply supported beam [18] with 15 similar elements, 16 nodes, and 30 DOFs, as shown in Figure 2. Increasing the number of divided elements facilitates accurate detection of damages; however, more sensors are still required for the derivation of mode shapes.

The simulated beam is considered as a concrete beam where $E=28 \mathrm{GPA}$ and $\rho=2500 \mathrm{~kg} / \mathrm{m}^{3}$.

\subsection{Investigation of the proposed method without noise}

In this study, damages were simulated by reducing the elasticity modulus. As shown in Table 2, different damage scenarios and damage severities were employed to examine the proposed method. A parametric study was carried out in order to investigate the convergence process for DS2 pattern in the simply supported beam. Different possible states of $\alpha_{4}$ and $\alpha_{6}$ parameters were studied with the accuracy of $1 \%$. As shown in Figure 3, the IGA converged into the exact values only after few generations, and in case the elements suspected of damages were known, 900 analyses were required to find accurate answers.

The convergence process of the objective function of the numerical example is shown in Figure 4 that contains two curves: "maximum population fitness" and "mean population fitness".

The "maximum population fitness" curve indicates the performance of the best chromosome in each
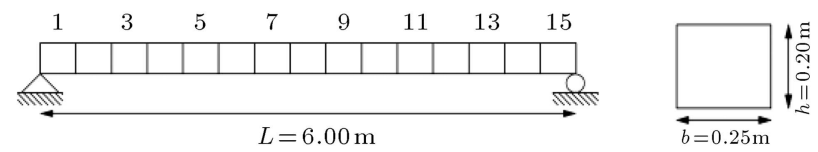

Figure 2. A simply supported beam with 15 elements.
Table 2. Damage scenarios of the simply supported beam.

\begin{tabular}{ccll}
\hline \multicolumn{2}{c}{ Damage scenario } & $\begin{array}{c}\text { Damaged } \\
\text { element(s) }\end{array}$ & $\begin{array}{c}\text { Damage } \\
\text { severity }\end{array}$ \\
\hline DS1 & Single damage & No. 6 & $10 \%$ \\
DS2 & Double damage & No. 4 & $10 \%$ \\
& & No. 6 & $15 \%$ \\
DS3 & Triple damage & No. 4 & $10 \%$ \\
& & No. 6 & $15 \%$ \\
& & No. 11 & $20 \%$ \\
& & & \\
DS4 & Multiple damage & No. 4, No. 6 & $10 \%$ \\
& & No. 8, No. 11 & $15 \%$ \\
& & No. 12 & $20 \%$ \\
\hline
\end{tabular}

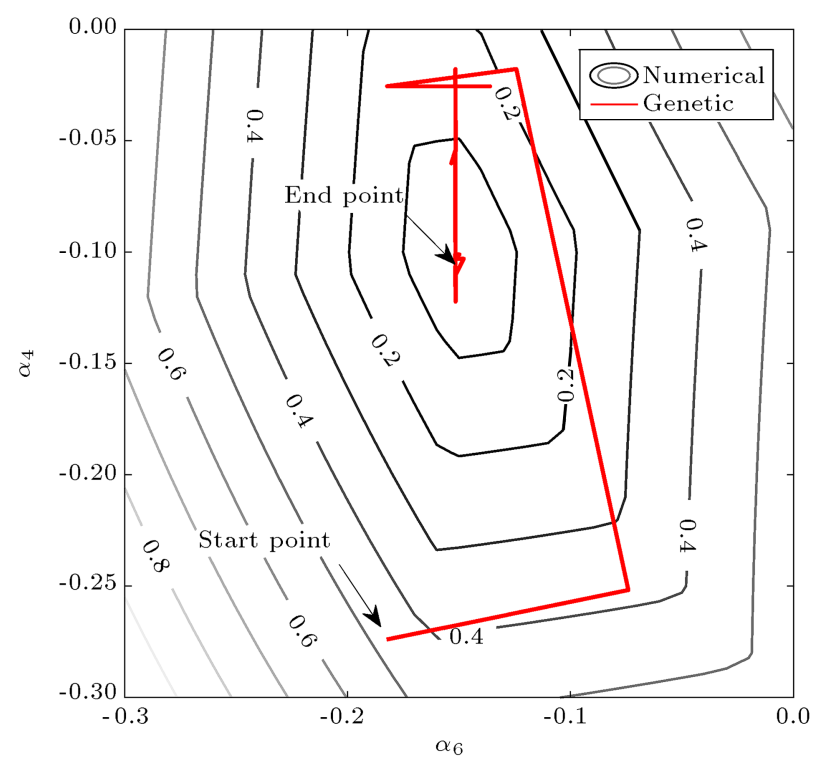

Figure 3. Parametric study of the stiffness reduction coefficient in DS2 pattern.

generation. As shown in these figures, in case the children chromosomes do not have better compatibility than the parent chromosomes, the curve becomes horizontal. This curve continues horizontally up until the improvement of one children chromosome compared with parent chromosomes. The best chromosome of each generation is directly transmitted to the next generation based on the elitism concept used in this algorithm. This idea prevents the distraction of improper children from the optimization path. The "mean population fitness" curve indicates the average compatibility of each generation determined based on the objective function. The elite chromosome transmitted from the prior generation is more likely to be 


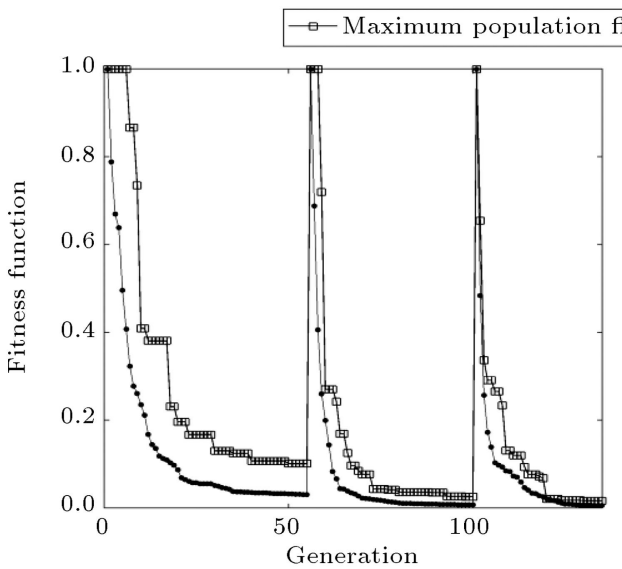

(a)

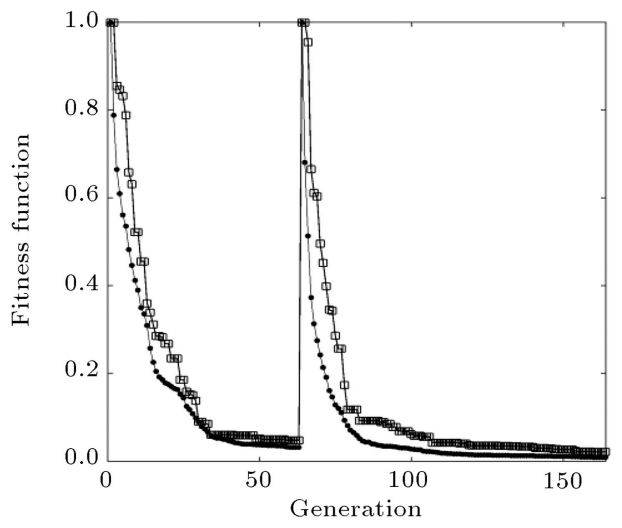

(c)

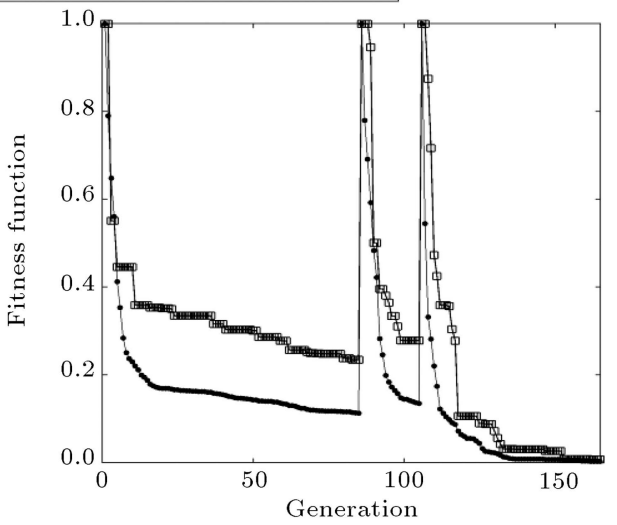

(b)

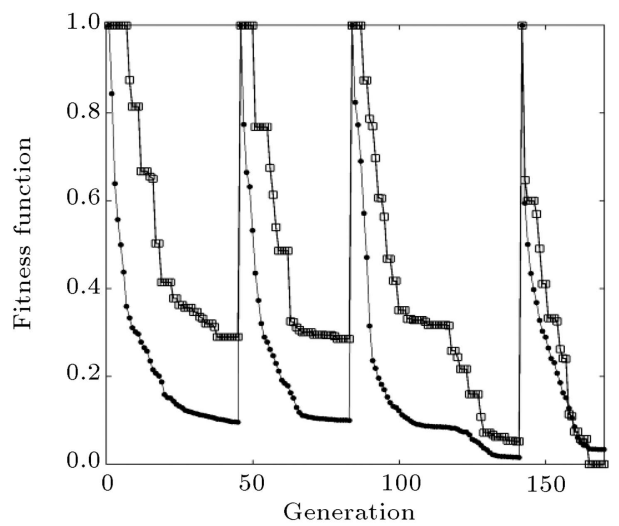

(d)

Figure 4. Convergence process of the simply supported beam: (a) DS1, (b) DS2, (c) DS3, and (d) DS4.

selected for reproduction than other chromosomes, and it can create children chromosomes with a high shared similarity with the parent chromosomes. The fitness values shown in this graph follow a descending trend.

According to Figure 4, all elements are added to the IGA for damage detection as inputs. Following a temporary convergence, the algorithm recognizes that the objective function is enhanced upon reducing the damage coefficients of some elements. Therefore, zero values are assigned to these elements and are removed from the problem space. This process decreases the number of variables and increases the accuracy of damage estimations. This cycle is repeated as long as the objective function reaches zero. Finally, the IGA determines the damage severity of elements with the highest accuracy. Since the process of damage detection starts randomly, it can challenge the time of achieving accurate damage coefficients; in other words, there is a possibility for the estimated damage coefficients at the first step to be similar to/different from the actual values. As shown in Figure 4, DS1 and DS2 patterns converge to optimal values after three iterations of IGA and elimination of healthy elements when DS3 and DS4 minimize the objective functions through 2 and 4 iterations, respectively.
However, it should be noted that the complexity of the examined structure or sophisticated damage scenario can affect the number of IGA iterations in finding a definite answer; therefore, the IGA removes fewer elements from the search space in each iteration and the search space enlarges at a lower speed; yet, it does not have any influence on the accuracy of damage detections.

Element damage severities of the simply supported beam are shown in Figure 5. According to this figure, in case there is no noise in the mode shape data, the damage severity is accurately calculated by only one mode (the first mode) with no errors after a few steps. In addition, some healthy elements in this figure are detected as slightly damaged elements at the first step of IGA implementation. Furthermore, the estimated damage coefficients of the damaged elements are subject to slight error. Through elimination of the number of healthy elements detected at the previous step, the damage coefficients of the remaining elements are identified with higher accuracy. As shown in Figure 5, some elements, slightly damaged with small damage coefficients at the early steps, are identified as the healthy elements and eliminated from the problem space at the next steps. Although some elements are 


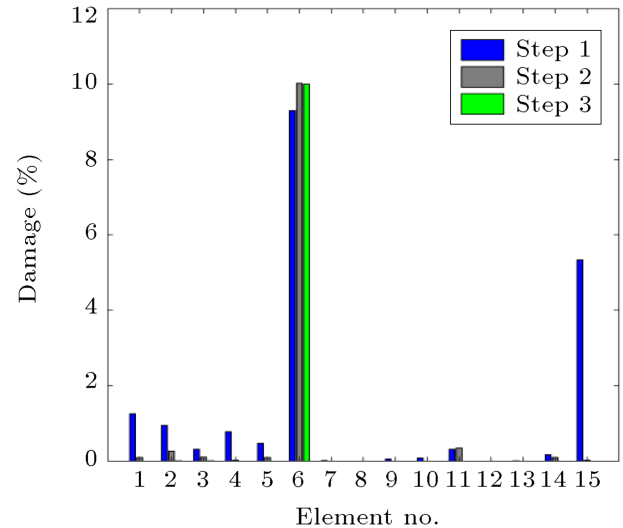

(a)

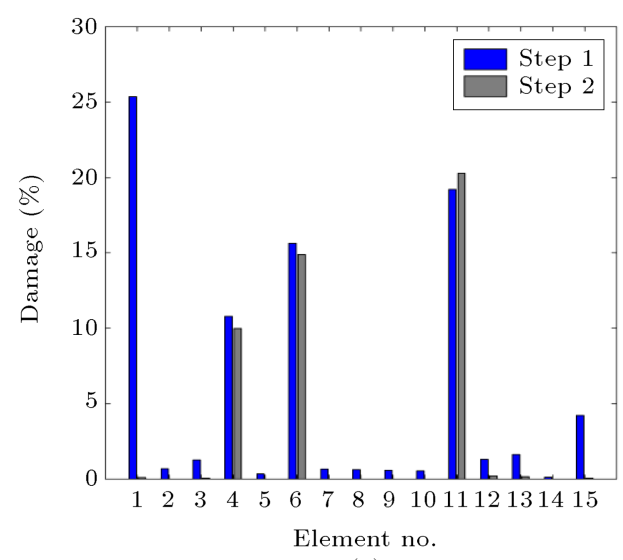

(c)

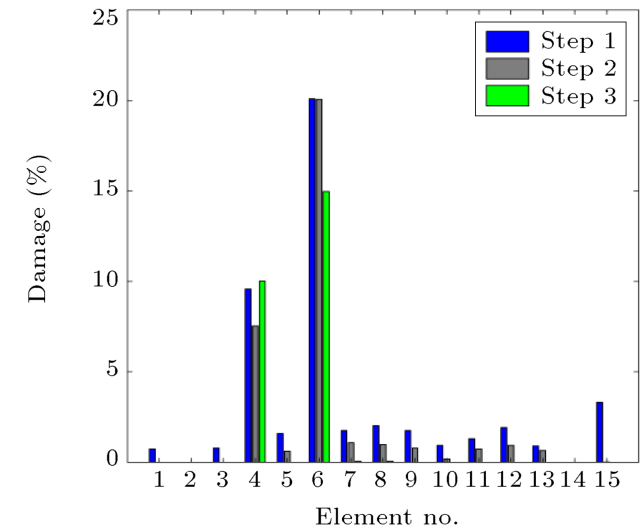

(b)

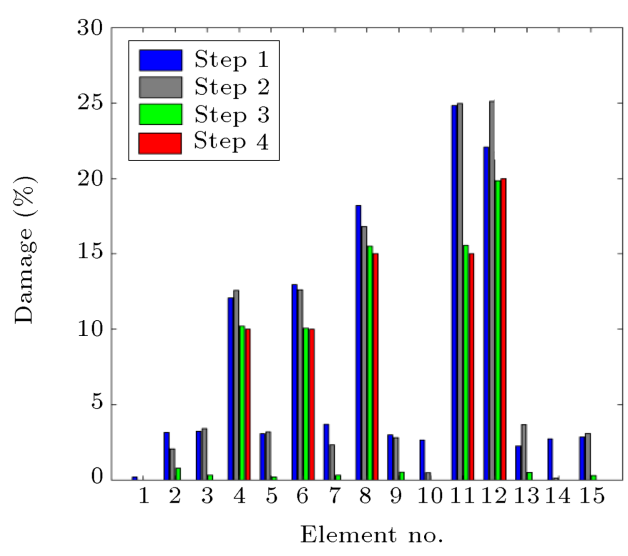

(d)

Figure 5. Damage severity of the simply supported beam in (a) DS1, (b) DS2, (c) DS3, and (d) DS4.

detected to be damaged at the early steps, their exact values can be reached.

\subsection{Investigation of the proposed method with noise}

In the estimation process of the information related to the damaged structures, complete noise elimination is impossible. In fact, the presence of noise in the obtained information from sensors causes a deviation from the exact values of damage severity. In this section, the sensitivity of the proposed method to the presence of noise in the data is investigated. In order to simulate the effect of noise, the mode shape data with different noise levels of $0.05 \%, 1 \%, 2 \%, 3 \%, 4 \%$, and $5 \%$ are contaminated. The noise-contaminated mode shapes are obtained through the following equation:

$$
\bar{\Phi}_{i r}=\Phi_{i r}\left(1+\gamma_{r}^{\Phi} \rho^{\Phi}\left|\Phi_{\max , i}\right|\right),
$$

where $\bar{\Phi}_{i r}$ and $\Phi_{i r}$ are the $i$ th mode shape components of $r$ th DOF with and without noise, respectively; and $\gamma_{r}^{\Phi}$ is a Gaussian random number with the mean and variance of zero and one, respectively; further, $\rho^{\Phi}$ is the noise level and $\left|\Phi_{\max , i}\right|$ is the $i$ th largest mode shape component without noise. The Coefficient of Variance (COV) is used here as a tool for investigating the damage estimation accuracy through the following equation:

$$
C O V=\frac{\sigma_{\alpha}}{\bar{\alpha}}
$$

where $\sigma_{\alpha}$ and $\bar{\alpha}$ are the standard deviation and average of the damaged coefficients, respectively, estimated by IGA through 1000 tests. In Figure 6, the average damage coefficients in different scenarios for the simply supported beam are shown in the presence of $1 \%$ noise in mode shapes. As shown in this figure, damages in healthy elements are approximately $1 \%$. Since the IGA which is based on mode shapes and is polluted by noises minimizes the objective function, these detections are not indicative of its poor performance. In other words, the IGA takes into account the mode shape variations because of the similarity between the noises to the damages.

Figure 7 shows the COV at different noise levels and in damaged scenarios. The results indicated that IGA could appropriately identify damage severities up to $1 \%$ noise level.

Given that the objective function of this algorithm is calculated using Eq. (13), which is directly affected by vibration mode shapes, the performance of 


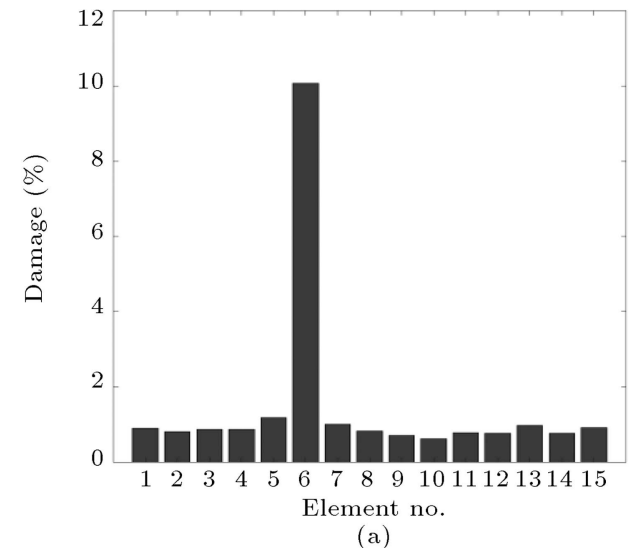

(a)

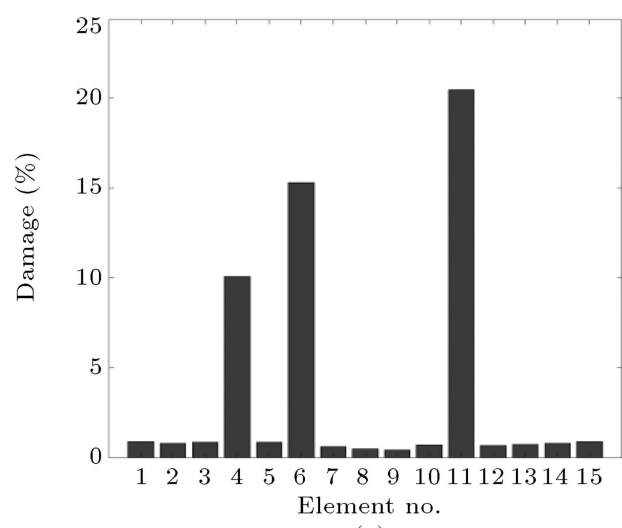

(c)

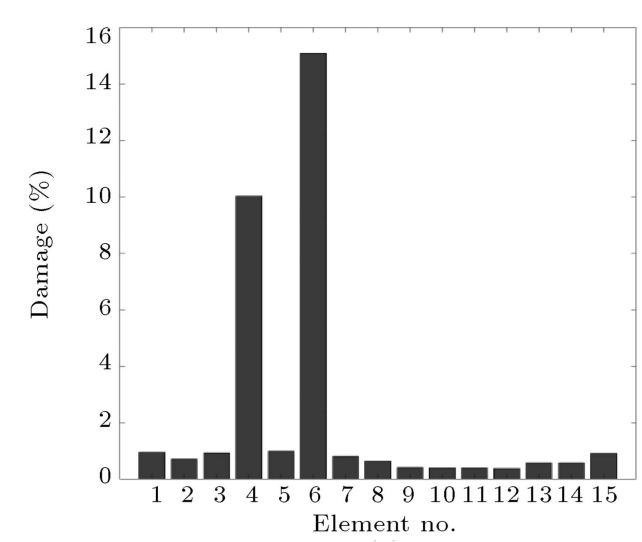

(b)

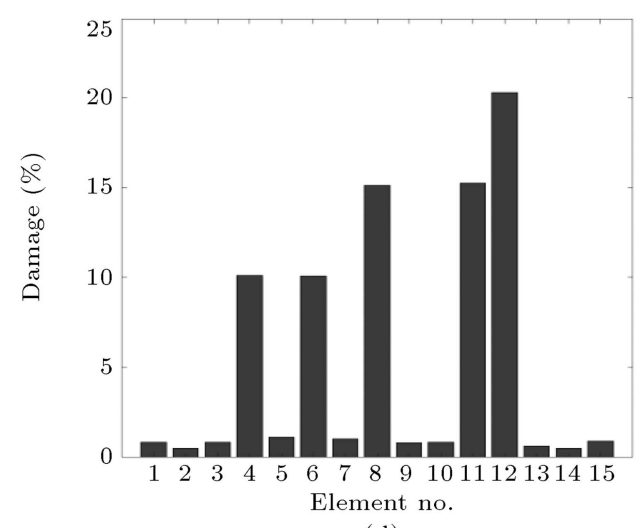

(d)

Figure 6. Damage averages of simply supported beam elements in (a) DS1, (b) DS2, (c) DS3, and (d) DS4 (with $1 \%$ noise).

the IGA will decrease upon increasing noise levels.

$$
\begin{aligned}
\Delta M S E_{i j}= & \frac{1}{2}\left\{\Phi_{i}^{d}\right\}^{T}[K]_{j}\left\{\Phi_{i}^{d}\right\}-\frac{1}{2}\left\{\Phi_{i}^{p d}\right\}^{T} \\
& {[K]_{j}\left\{\Phi_{i}^{p d}\right\} . }
\end{aligned}
$$

In case of employing pure data, the proposed method can exactly identify damage severities. Therefore, by replacing the objective functions that are not affected by the noise, the performance of IGA can be improved in the absence/presence of noise and only through the first mode.

\section{Experimental validation}

An experimental setup was studied to investigate whether the application of rational DOFs could affect the proposed method and to help identify the location and severity of damages through only one mode. The specific objective of this example was to identify the damage location and severity using only one mode. In order to validate the performance of the proposed method, a simply supported steel beam including 16 segments of $7.5 \mathrm{~cm}$ was investigated. The mechanical and geometrical properties of the beam are listed in Table 3. Since the mass of each acceleration sensor, the wire connected to it, and Plexiglass piece on which the sensor was placed were about 30 grams, moving the sensors did not change the mass distribution and thus, equivalent added masses were used in other places with no such sensors. As shown in Table 3, the density of the beam was considered to be higher than that of the current steel elements mainly because the element markers and accelerometers used for data acquisition

Table 3. Properties of the simply supported beam.

\begin{tabular}{ccccc}
\hline $\begin{array}{c}\text { Young's modulus } \\
\left(\mathbf{N} / \mathbf{m}^{\mathbf{2}}\right)\end{array}$ & $\begin{array}{c}\text { Density } \\
\left(\mathbf{k g} / \mathbf{m}^{\mathbf{3}}\right)\end{array}$ & $\begin{array}{c}\text { Length } \\
\mathbf{( \mathbf { m m } )}\end{array}$ & $\begin{array}{c}\text { Width } \\
(\mathbf{m m})\end{array}$ & $\begin{array}{c}\text { Thickness } \\
(\mathbf{m m})\end{array}$ \\
\hline $2 \times 10^{11}$ & 11266 & 1200 & 83 & 3 \\
\hline
\end{tabular}




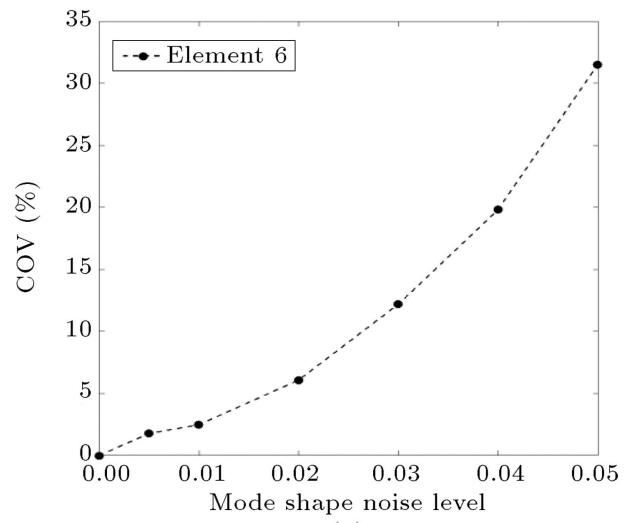

(a)

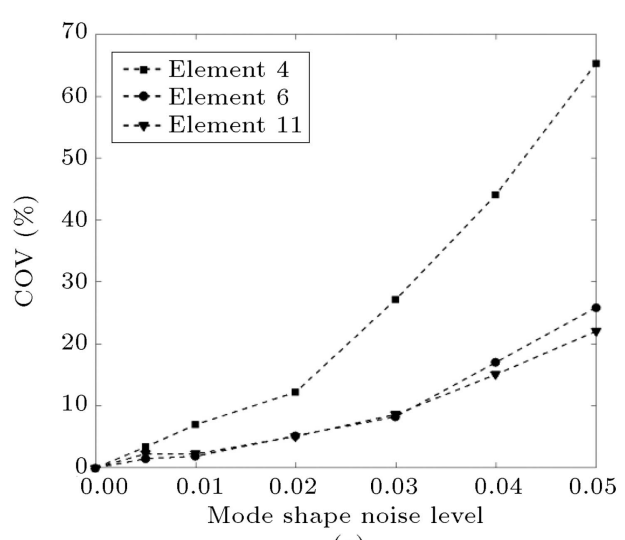

(c)

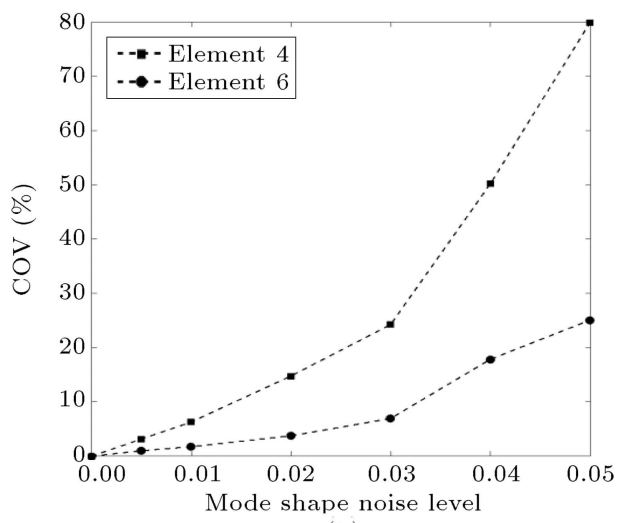

(b)

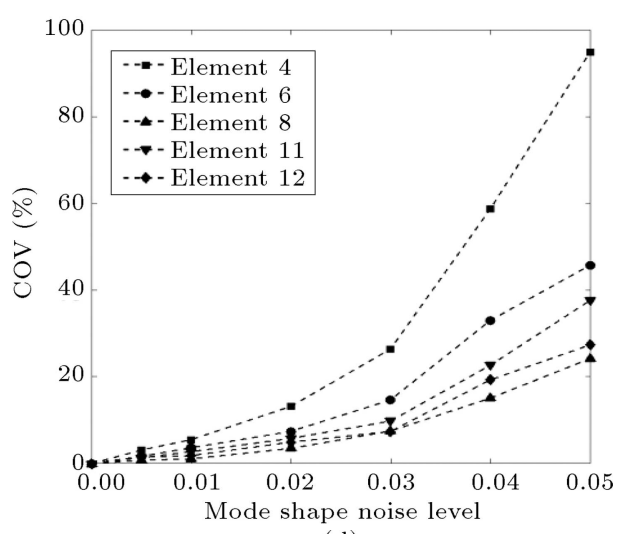

(d)

Figure 7. Coefficients of variance for the simply supported beam in (a) DS1, (b) DS2, (c) DS3, and (d) DS4.

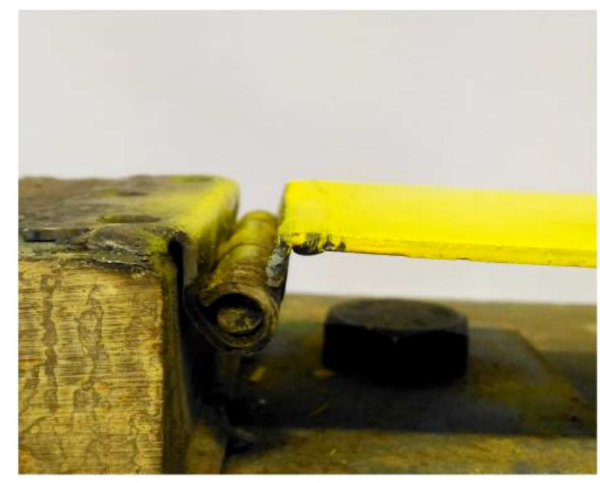

(a)

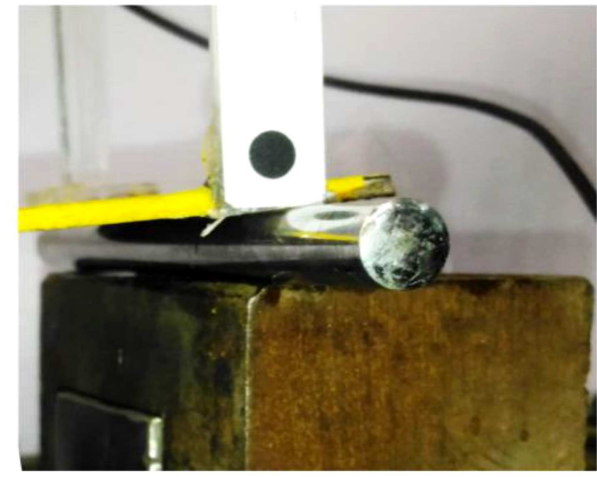

(b)

Figure 8. Experimental modeling of supports: (a) The joint support and (b) the roller support.

as well as the added masses were more significant than the beam. Therefore, the mass of these attachments was distributed across the total length of the beam. The hinge and roller supports were constructed similar to theoretical examples, as shown in Figure 8.

The overall view of the beam and measuring instruments including accelerometers, markers, and computer system equipped with data logger and camera are shown in Figure 9. For this model, two different damage scenarios were considered. The first scenario includes a $30 \%$ stiffness reduction in the 6 th element.
In the second scenario, in addition to the element number 6 , a $20 \%$ stiffness reduction was also considered for the 13th element. The transverse cuts were exerted to the desired locations while the measurable damages continued throughout the distance between the two markers. Given that one of the assumptions of this method, before and after the damage, was that the mass was constant, the cut pieces were again stuck to the beam so that the mass would remain unchanged without increasing the stiffness. Figure 10 shows how the damages are incurred against the predetermined 


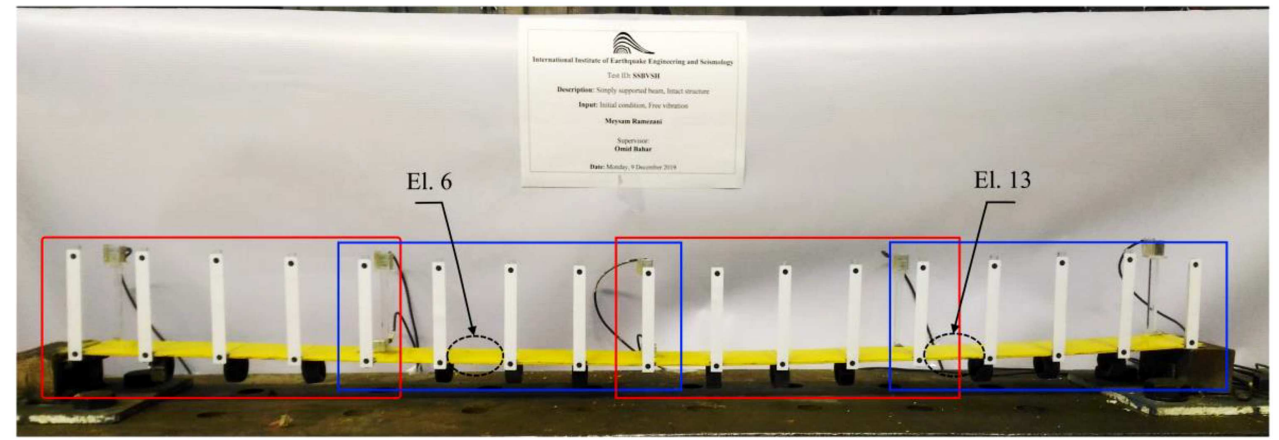

(a)

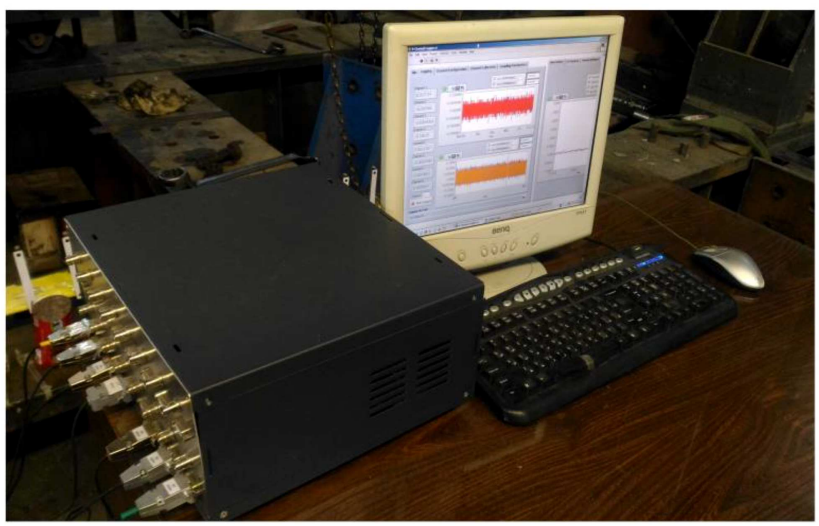

(b)

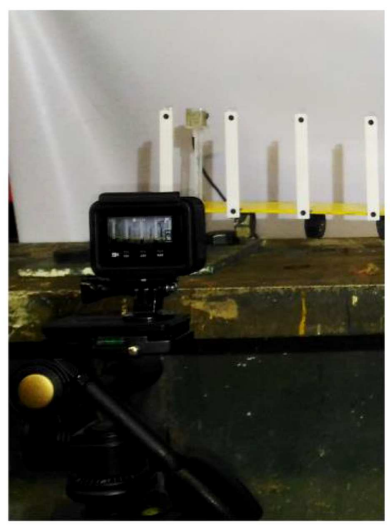

(c)

Figure 9. (a) The overall view of the beam and division of the filming range. (b) Computer system with data logger. (c) Video camera.

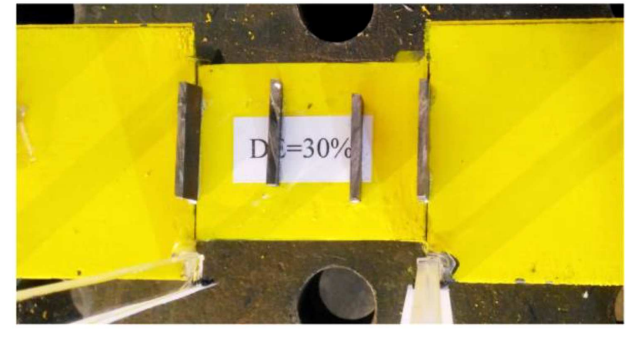

(a)

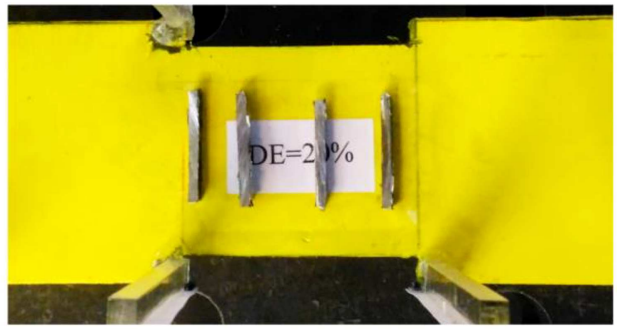

(b)

Figure 10. Details of the damaged elements: (a) Element 6 and (b) element 13.

elements based on Scenarios 1 and 2. Of note, the damage severity of elements was equal to the ratio of the moment of inertia of the reduced and intact sections. Since the constructed beam has no offplane motion, it can be referred to as a $2 \mathrm{D}$ model. On the contrary, because the horizontal stiffness of the beam was higher than the vertical and rotational stiffness, these DOFs were ignored in the numerical model updating. The data required for this method contain the first mode shape and frequency, and there are different methods for extracting this information from the structural response. For this reason, a static load was applied to the middle of the beam and then, it was suddenly removed to create free vibrations. In this study, the displacement and acceleration responses in both vertical and rotational directions were obtained at defined points under free vibration using a visionbased method and uniaxial accelerometer sensors, respectively. These responses were then used to obtain the vertical and rotational mode shapes via Fourier analysis. The output of these two methods would be both first-mode frequency and first-mode shape vector containing the mode shapes of both rotational and vertical DOFs.

\subsection{Vision-based method for data acquisition}

The mechanism of measuring the movement in the vision-based method involves installing cameras on a tripod, setting tracking targets, using template matching algorithm, and converting the coordinates. For 


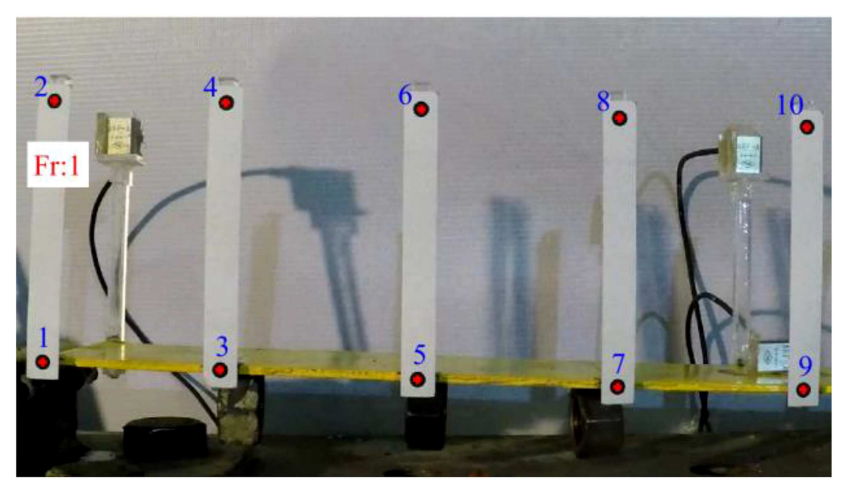

Figure 11. Detecting the center of the circles.

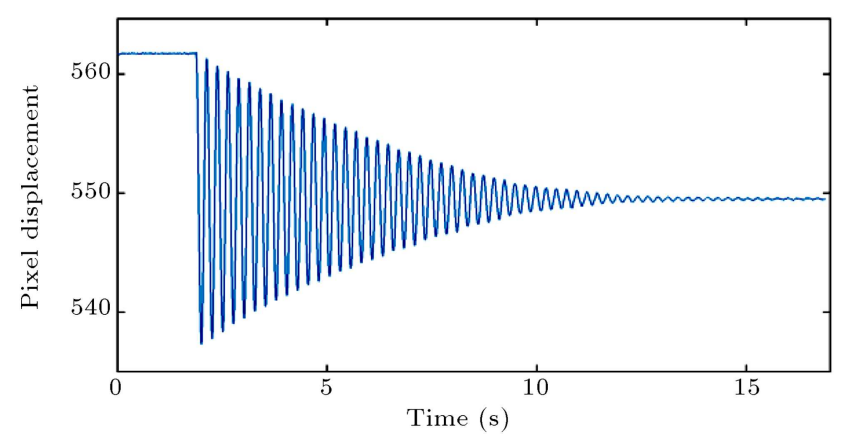

Figure 12. Vertical pixel displacement of point 9.

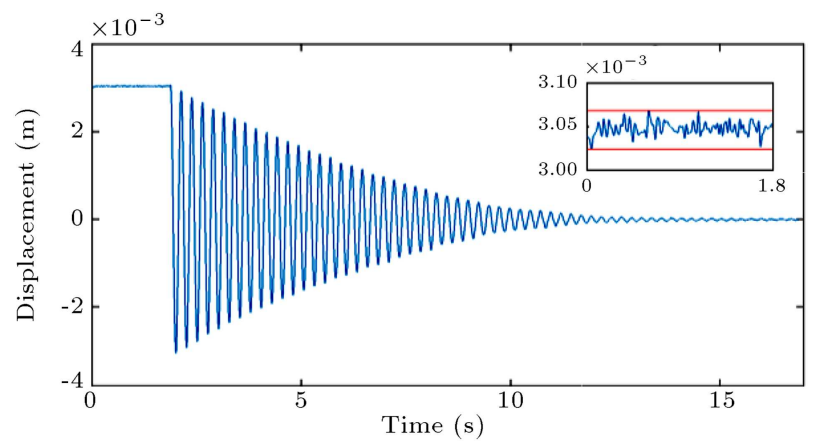

Figure 13. Vertical displacement of point 9.

this purpose, a camera capable of recording video in 240 frames per second was used. To increase the resolution of the images, filming was done at four stages from different parts of the beam, as shown in Figure 9(a). Black circles on a white background were used as the targets. These targets were stuck on a rigid and negligible mass element called marker with Plexiglass material. By detecting the center of the circles in each frame, the movement signal was obtained based on the position of the image pixels. In order to achieve physical and real displacement, a relationship was established between pixel coordinates and real coordinates. The steps are illustrated in Figures 11 to 13. As shown in Figure 13, the recorded noise is approximately $2.2 \times 10^{-5} \mathrm{~m}$, which can be decreased or increased by changing the distance between the camera and the target.
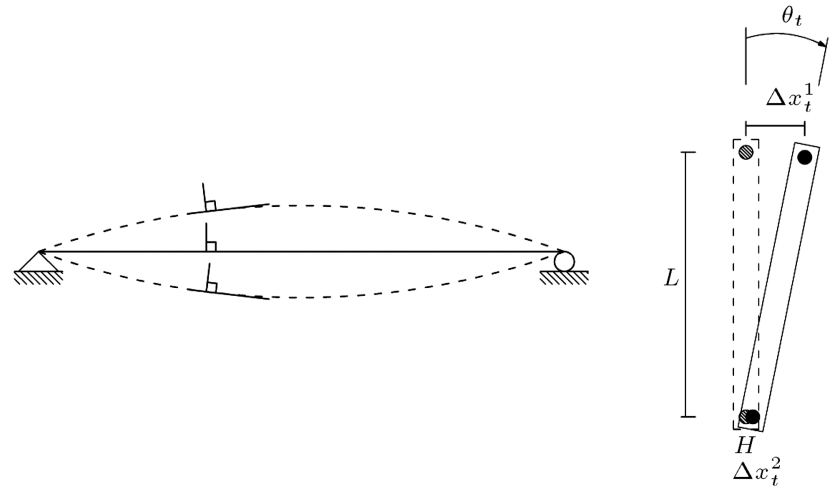

Figure 14. Vibration of the beam and the targets installed on it.

As discussed in the previous section, the vertical and rotational mode shapes were obtained to detect damages using only one mode. The most significant factor that ensures the success of this process is the information used as the inputs such as rotational mode shapes. Figure 14 shows how the targets move during the vibration. If a rigid connection is made between the marker and the beam, no relative rotation will occur and the rotation of the marker will be equal to that of the beam at that location. Based on Eq. (14), by tracking the relative horizontal displacement in markers, the rotation of the beam can be obtained wherever the marker is located.

$$
\theta_{t}=\operatorname{ArcSin}\left(\frac{\Delta x_{t}^{1}-\Delta x_{t}^{2}}{L}\right) \simeq\left(\frac{\Delta x_{t}^{1}-\Delta x_{t}^{2}}{L}\right),
$$

where $\theta_{t}$ is the time history of rotation; $\Delta x_{t}^{1}$ and $\Delta x_{t}^{2}$ are the time histories of horizontal displacement of the targets; and $L$ is the distance from the center of two circles.

A comparison of the mode shapes obtained from the experimental signals and numerical modeling indicated some slight discrepancies caused by scaling each part of the mode shapes or local imperfections in the beam. To reduce the effect of the scaling errors, the mode shapes were obtained using the same method and reference scale in all scenarios. These differences were then added to the experimental mode shapes in all scenarios as bias values.

The first mode shape of the damaged beam acquired by the experimental data is presented in Figures 15 and 16. A comparison of the rotational mode shapes of both healthy and damaged beams in Figure 15(b) revealed that the rotational mode shape of the damaged beam passed exactly between points 6 and 7 , represented as element 6 , through the rotational mode shape of the healthy beam. According to Figure 16(b), the rotational mode shape of the damaged beam passed through that of the healthy beam in elements 6 and 13 . In this respect, it can 


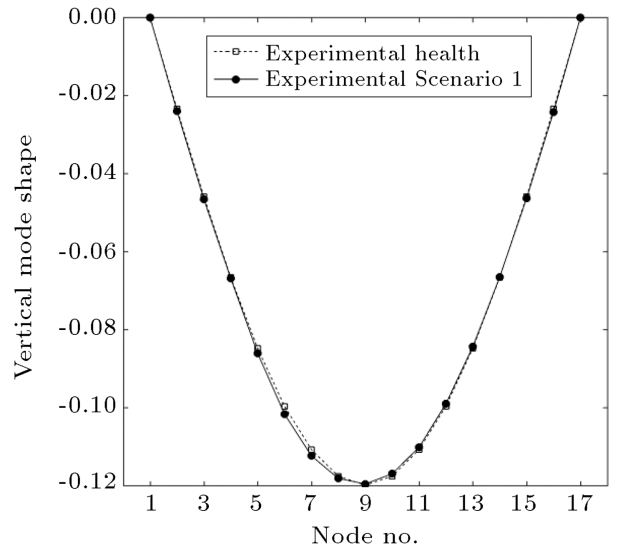

(a)

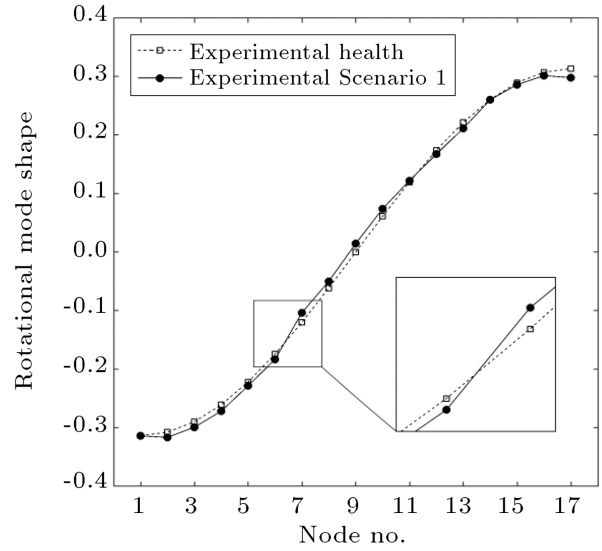

(b)

Figure 15. First mode shape (Scenario 1): (a) Vertical mode shape and (b) rotational mode shape.

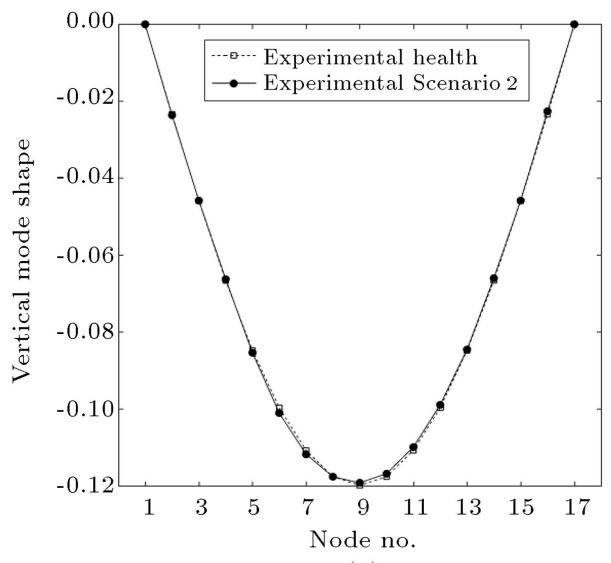

(a)

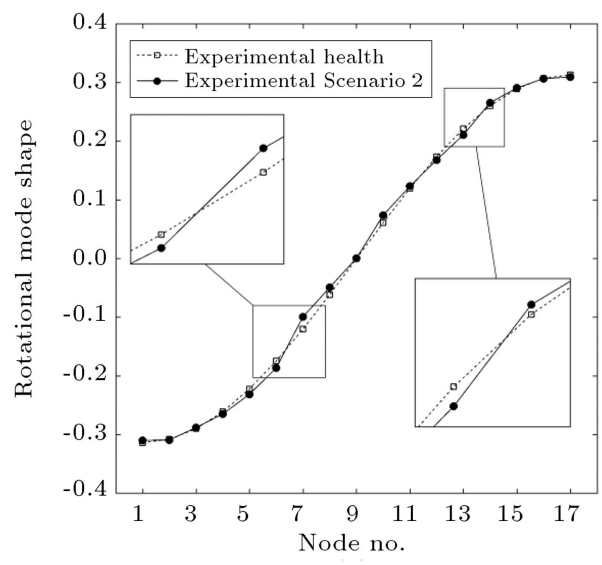

(b)

Figure 16. First mode shape (Scenario 2): (a) Vertical mode shape and (b) rotational mode shape.

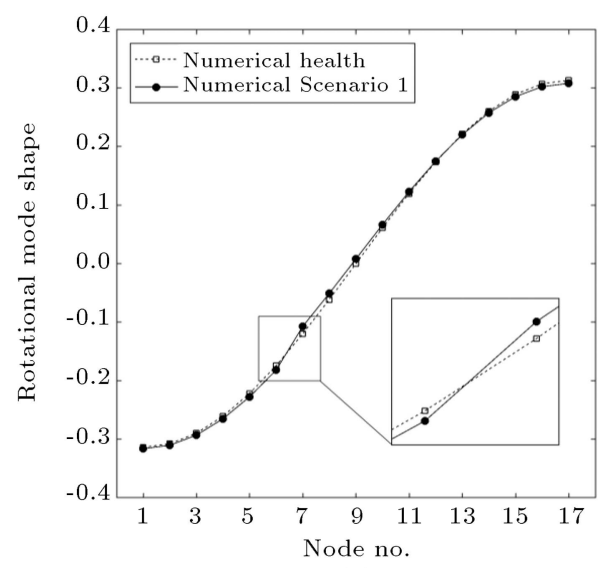

(a)

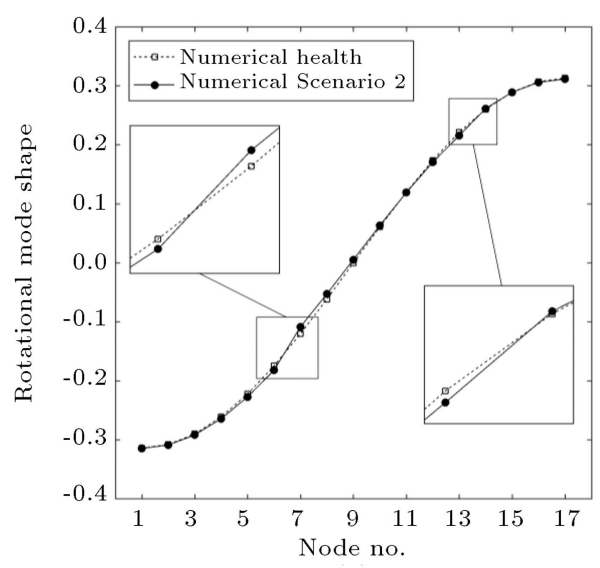

(b)

Figure 17. First rotational mode shape: (a) Scenario 1 and (b) Scenario 2.

be concluded that the intersection of two lines in the rotational mode shape pointed to the location of the damage. According to Figure 17, a comparison of the rotational mode shapes obtained from the numerical results confirmed this finding.

Comparison of the vertical mode shapes in both scenarios failed to provide any specific information about the damaged elements. This is the reason why many researchers prefer using higher modes up until identification of the damaged elements.

Some researchers have employed the first derivative of the transition mode to identify damages [37]. As 


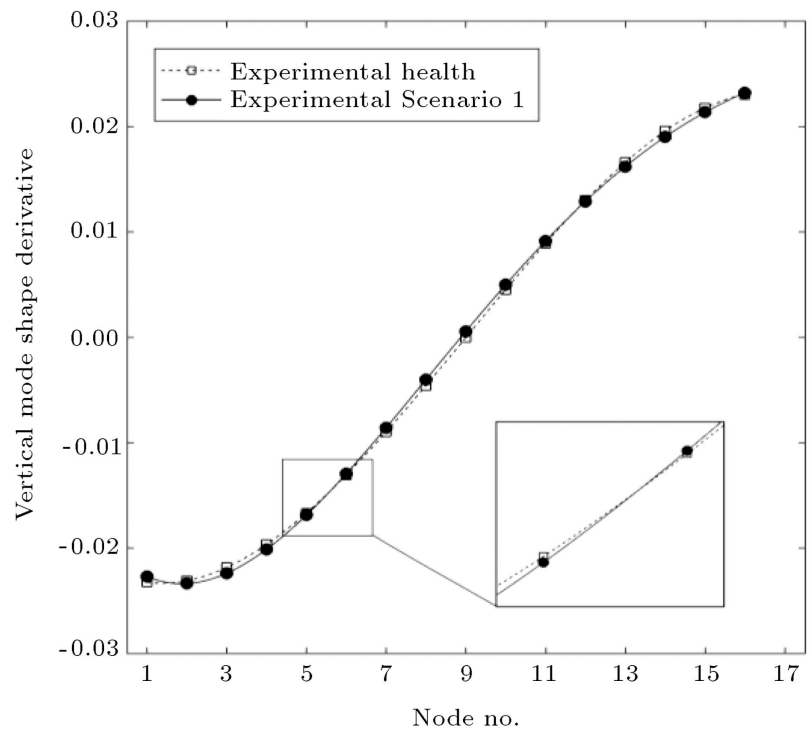

Figure 18. The first derivative of the vertical model shape based on experimental results.

shown in Figure 18, the derivative of the experimental vertical mode shape of the healthy and damaged structures (Scenario 1) was employed to prove that the rotational mode shape obtained using the proposed method could provide more complete and accurate information about the damage. In this figure, the rotational mode shapes intersect at the 5 th element, indicating that the 5 th element is mistakenly identified as the damaged element. This is the reason why direct methods should be utilized to extract rotational mode shapes instead of indirect methods such as calculating the derivative of the translational mode shapes. Figure 18 illustrates the derivation of the curve fit to the vertical mode shape.

In Figure 19, the damage severities of elements are shown in different steps. In Scenario 1, after three steps, respectively 9, 1 , and 1 element(s) was/were identified as healthy; however, the damage can be observed for all the elements in Scenario 2. Compared

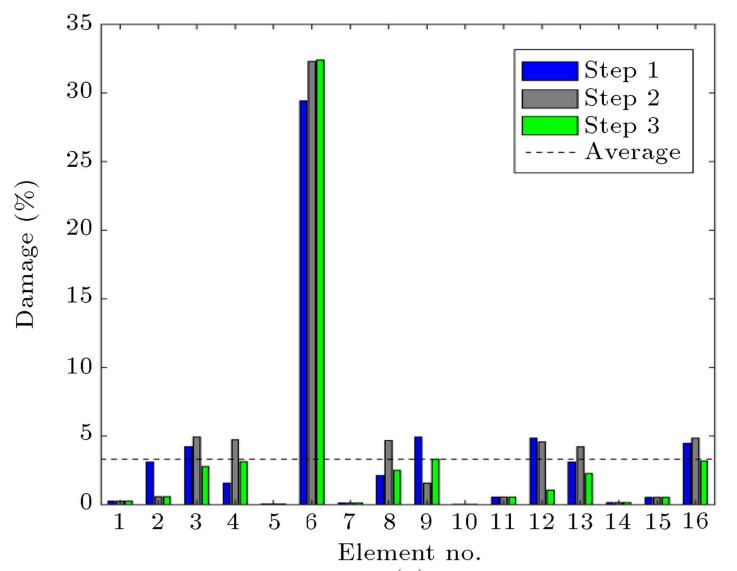

(a) to the numerical example in the previous section, the presence of noise in the data could significantly reduce the quality of the obtained modal information, thus decreasing the number of the identified healthy elements. However, as already mentioned in the comparison results of rotational mode shapes, despite the reduced quality of modal information, the location and severity of damages can be obtained with acceptable accuracy for both scenarios. In order to increase the reliability of the results of the identified damage, the average damage severities in both scenarios were determined, depicted by dashed line in Figure 19. In this figure, the damage severities below this line can be considered as unreliable results. It can be concluded that this method failed to determine the initial small damages.

\subsection{Accelerometer installation method for data acquisition}

Due to the limited number of accelerometers, eight sensors have been used including three and five sensors for vertical and rotational data acquisition, respectively. To measure the vertical DOFs, the sensors must be attached to the beam such that no relative acceleration is created between the sensors and the beam. The rotational DOFs were also obtained by recording the horizontal acceleration at a certain level of the beam. To this end, acceleration sensors were placed on Plexiglass pieces and then, firmly connected to the beam. However, these accelerometers record the acceleration in line with their horizontal axis, which is not exactly the same as the horizontal acceleration above the pieces because the horizontal axis of the accelerometers rotates when the beam vibrates. Given that the rotation is quite small, the recorded acceleration can be considered almost equal to the horizontal acceleration of the top of pieces. In addition, the weight of the sensors can create an inertial force which can cause errors in the rotational acceleration. Another issue that causes errors in data acquisition with accelerometers

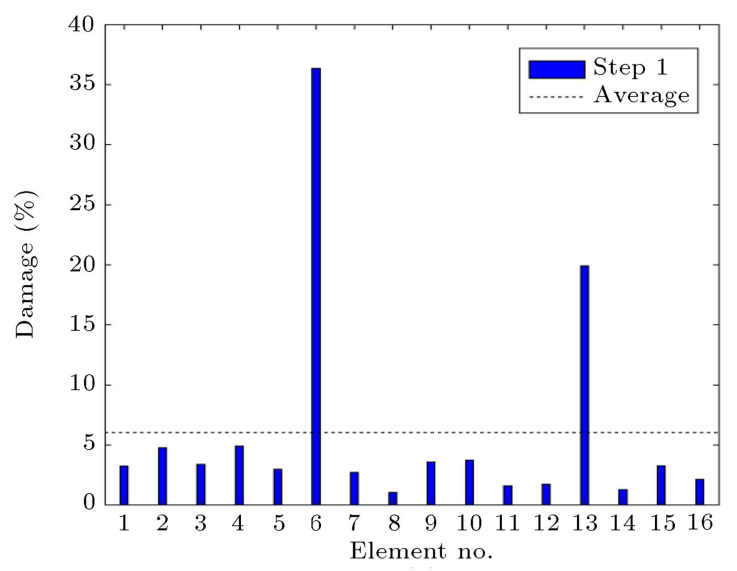

(b)

Figure 19. Identification of damage severities on different steps: (a) Scenario 1 and (b) Scenario 2. 


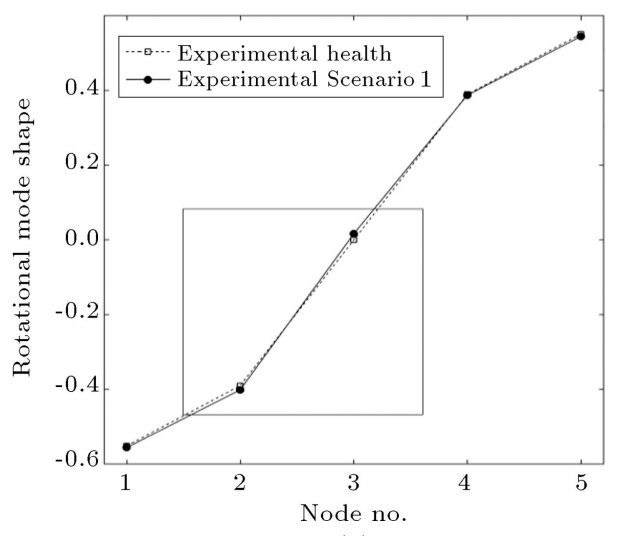

(a)

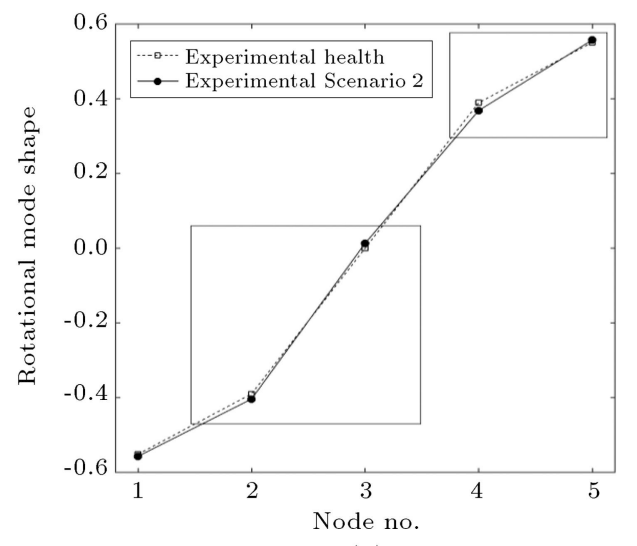

(b)

Figure 20. First rotational mode shape: (a) Scenario 1 and (b) Scenario 2.

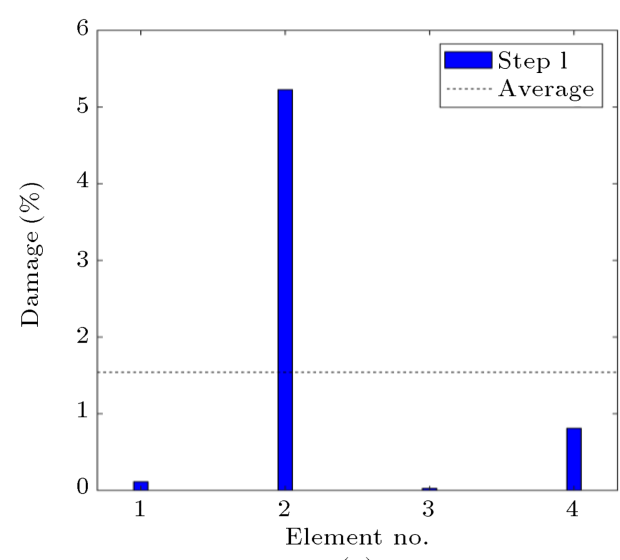

(a)

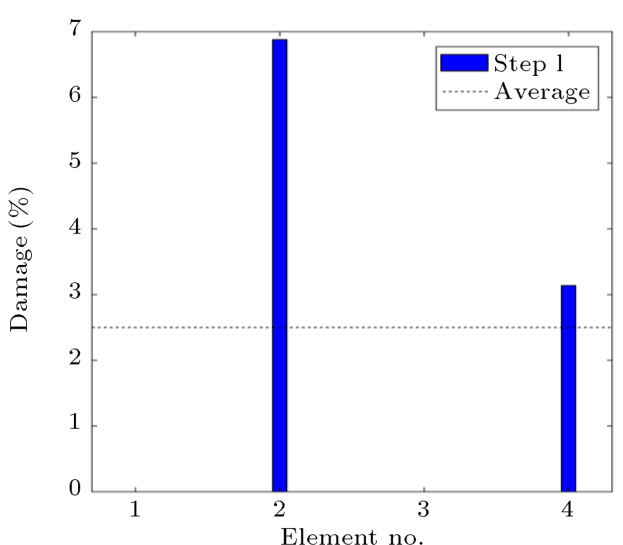

(b)

Figure 21. Identification of damage severities in different steps: (a) Scenario 1 and (b) Scenario 2.

is the reduced sensitivity of the sensors over time. Each accelerometer has a specific capacity that when accelerated more than its capacity, damages would occur. For this reason, the manual calibration method was employed because the calibration coefficients in the sensor catalog were not as sensitive as before. The rotational acceleration of each point of the beam can be calculated through Eq. (15):

$$
\ddot{\theta}_{t}=\operatorname{ArcSin}\left(\frac{\Delta \ddot{x}_{t}}{L}\right) \simeq\left(\frac{\Delta \ddot{x}_{t}}{L}\right),
$$

where $\Delta \ddot{x}_{t}$ denotes the time history of horizontal acceleration axis of the accelerometer and $L$ is the height of Plexiglass piece.

The first rotational mode shapes of the damaged beam are shown in Figure 20. Here, similar to the results obtained from the vision-based method, the rotational mode shapes of the healthy and damaged beams passed over each other in the location of the cut element.

Since only a part of the distance between these two sensors was cut, it would be impossible to determine the exact damage in each element. As a rough approximation, the moment of inertia for the damaged element can be calculated by the weighted arithmetic mean of the moment of inertia. This approximation is only valid until high-severity damage does not occur at a limited length. In case this assumption is accepted, the reduced stiffness rates in elements 2 and 4 are $7.5 \%$ and $5 \%$. The estimated damage severities are shown in Figure 21. As shown in this figure, the extent of the estimated damage is close to that of the calculated values; hence, the accuracy of the results can be ensured.

\section{Conclusion}

In this study, a new approach was proposed in which the least amount of information on vibration mode shapes, i.e., only the first mode, was required for damage detection. To this end, rotational mode shapes containing remarkable information about the location and severity of the damage were employed. This information was obtained using simple data-acquisition methods such as placing accelerometers and visionbased methods as well as geometrical formulations. 
After extracting the first mode using the proposed method, damage coefficients were obtained by eliminating the healthy elements indirectly. This type of attitude in obtaining the damage coefficients would form a robust algorithm.

Numerical and experimental studies on a simply supported beam were carried out. The feasibility and reliability of this method were studied using the first mode shape. The results of the numerical example showed that the proposed algorithm could exactly detect damage severity without noise, only using the first mode information. Furthermore, several simulations were performed to investigate the performance of the proposed method in the presence of noise. In order to simulate the effect of noise, the mode shape data were contaminated at different noise levels $(0.5 \%, 1 \%, 2 \%$, $3 \%, 4 \%$, and $5 \%$ ). The results demonstrated that this method had acceptable performance in determining damage severities for the noise levels up to $1 \%$. Experimental results demonstrated that damage location and severity estimation could be successfully detected using only the first mode of vibration in an acceptable range and despite the presence/absence of modal information at both ends of the cut beam. In summary, the results showed that the rotational Degrees Of Freedom (DOFs) played a key role in detecting the location and severity of damages.

\section{Acknowledgement}

The present study is a part of the first author's PhD thesis at the International Institute of Earthquake Engineering and Seismology, whose support is gratefully appreciated.

\section{References}

1. Günay, S., Mosalam, K.M., Archbold, J., Dilsiz, A., DJIMA, W., Gupta, A., Javadinasab Hormozabad, S., Hassan, W., Heresi, P., Morales-Beltran, M., Muin, S., Robertson, I., Romão, X., and Kijewski-Correa, T., Preliminary Virtual Reconnaissance Report (PVRR), DesignSafe-CI (2020).

2. Roueche, D., Robertson, I., Prevatt, D., Mosalam, K., Kijewski-Correa, T., Cortes, M., Gutierrez Soto, M., and Javadinasab Hormozabad, S., StEER-Hurricane Eta, DesignSafe-CI (2020).

3. Doebling, S.W., Farrar, C.R., and Prime, M.B. "A summary review of vibration-based damage identification methods", Shock and Vibration Digest, 30(2), pp. 91-105 (1998).

4. Whalen, T.M. "The behavior of higher order mode shape derivatives in damaged, beam-like structures", Journal of Sound and Vibration, 309(3-5), pp. 426464 (2008).

5. Duan, Z., Spencer, B., Yan, G., and Ou, J. "An improved optimal elemental method for updating finite element models", EEEV, 3(1), pp. 67-74 (2004).

6. Gao, Y. and Spencer, B. "Damage localization under ambient vibration using changes in flexibility", Earthquake Engineering and Engineering Vibration, 1(1), pp. 136-144 (2002).

7. Pandey, A. and Biswas, M. "Damage detection in structures using changes in flexibility", Journal of Sound and Vibration, 169(1), pp. 3-17 (1994).

8. Pandey, A., Biswas, M., and Samman, M. "Damage detection from changes in curvature mode shapes", Journal of Sound and Vibration, 145(2), pp. 321-332 (1991).

9. Shi, Z., Law, S., and Zhang, L. "Structural damage localization from modal strain energy change", Journal of Sound and Vibration, 218(5), pp. 825-844 (1998).

10. Qi, G., Xun, G., Xiaozhai, Q., Dong, W., and Chang, P. "Local measurement for structural health monitoring", Earthquake Engineering and Engineering Vibration, 4(1), pp. 165-172 (2005).

11. Yang, Q. and Liu, J. "Damage identification by the eigenparameter decomposition of structural flexibility change", International Journal for Numerical Methods in Engineering, 78(4), pp. 444-459 (2009).

12. Li, J., Wu, B., Zeng, Q., and Lim, C.W. "A generalized flexibility matrix based approach for structural damage detection", Journal of Sound and Vibration, 329(22), pp. 4583-4587 (2010).

13. Shi, Z., Law, S., and Zhang, L.M. "Structural damage detection from modal strain energy change", Journal of Engineering Mechanics, 126(12), pp. 1216-1223 (2000).

14. 14. Moradipour, P., Chan, T.H., and Gallage, C. "An improved modal strain energy method for structural damage detection, 2D simulation", Structural Engineering and Mechanics, 54(1), pp. 105-119 (2015).

15. Homaei, F., Shojaee, S., and Amiri, G.G. "A direct damage detection method using multiple damage localization index based on mode shapes criterion", Struct. Eng. Mech., 49(2), pp. 183-202 (2014).

16. Yan, W.-J. and Ren, W.-X. "Closed-form modal flexibility sensitivity and its application to structural damage detection without modal truncation error", Journal of Vibration and Control, 20(12), pp. 18161830 (2014).

17. Yazdanpanah1a, O. and Seyedpoor, S. "A new damage detection indicator for beams based on mode shape data", Structural Engineering and Mechanics, 53(4), pp. 725-744 (2015).

18. Shahri, A.H. and Ghorbani-Tanha, A. "Damage detection via closed-form sensitivity matrix of modal kinetic energy change ratio", Journal of Sound and Vibration, 401, pp. 268-281 (2017).

19. Gomes, H. and Silva, N. "Some comparisons for damage detection on structures using genetic algorithms and modal sensitivity method", Applied Mathematical Modelling, 32(11), pp. 2216-2232 (2008). 
20. Seyedpoor, S. "A two stage method for structural damage detection using a modal strain energy based index and particle swarm optimization", International Journal of Non-Linear Mechanics, 47(1), pp. 1-8 (2012).

21. Xu, H., Ding, Z., Lu, Z., and Liu, J. "Structural damage detection based on chaotic artificial bee colony algorithm", Struct. Eng. Mech., 55(6), pp. 1223-1239 (2015).

22. Kaveh, A. and Zolghadr, A. "Guided modal strain energy-based approach for structural damage identification using tug-of-war optimization algorithm", Journal of Computing in Civil Engineering, 31(4), p. 04017016 (2017).

23. Seyedpoor, S.M., Norouzi, E., and Ghasemi, S. "Structural damage detection using a multi-stage improved differential evolution algorithm (numerical and experimental)", Smart Structures and Systems, 21(2), pp. $235-248$ (2018).

24. Kaveh, A., Hoseini Vaez, S., and Hosseini, P. "Enhanced vibrating particles system algorithm for damage identification of truss structures", Scientia Iranica, 26(1), pp. 246-256 (2019).

25. Kaveh, A., Vaez, S.H., Hosseini, P., and Fathali, M. "A new two-phase method for damage detection in skeletal structures", Iranian Journal of Science and Technology, Transactions of Civil Engineering, 43(1), pp. 49-65 (2019).

26. Nobahari, M. and Seyedpoor, S. "Structural damage detection using an efficient correlation-based index and a modified genetic algorithm", Mathematical and Computer Modelling, 53(9-10), pp. 1798-1809 (2011).

27. Toloue, I., Liew, M., and Hamonangan Harahap, I. "Damage detection in frame structures using noisy accelerometers and Damage Load Vectors (DLV)", Scientia Iranaica, International Journal of Science and Technology, 27(4), pp. 1776-1785 (2018).

28. Liang, Y., Feng, Q., Li, H., and Jiang, J. "Damage detection of shear buildings using frequency-change-ratio and model updating algorithm", Smart Structures and System, 23(2), pp. 107-122 (2019).

29. Khatir, S., Wahab, M.A., Boutchicha, D., and Khatir, T. "Structural health monitoring using modal strain energy damage indicator coupled with teachinglearning-based optimization algorithm and isogoemetric analysis", Journal of Sound and Vibration, 448, pp. 230-246 (2019).

30. Mishra, M., Barman, S.K., Maity, D., and Maiti, D.K. "Ant lion optimisation algorithm for structural damage detection using vibration data", Journal of Civil Structural Health Monitoring, 9(1), pp. 117-136 (2019).

31. Krishnanunni, C., Raj, R.S., Nandan, D., Midhun, C., Sajith, A., and Ameen, M. "Sensitivity-based damage detection algorithm for structures using vibration data", Journal of Civil Structural Health Monitoring, 9(1), pp. 137-151 (2019).
32. Alten, K., Ralbovsky, M., Vorwagner, A., Toplitzer, H., and Wittmann, S. "Evaluation of different monitoring techniques during damage infliction on structures", Procedia Engineering, 199, pp. 1840-1845 (2017).

33. Erdenebat, D., Waldmann, D., Scherbaum, F., and Teferle, N. "The Deformation Area Difference (DAD) method for condition assessment of reinforced structures", Engineering Structures, 155, pp. 315-329 (2018).

34. Hester, D., Brownjohn, J., Huseynov, F., Obrien, E., Gonzalez, A., and Casero, M. "Identifying damage in a bridge by analysing rotation response to a moving load", Structure and Infrastructure Engineering, 16(7), pp. 1050-1065 (2020).

35. Huseynov, F., Kim, C., OBrien, E., Brownjohn, J., Hester, D., and Chang, K. "Bridge damage detection using rotation measurements-experimental validation", Mechanical Systems and Signal Processing, 135, p. 106380 (2020).

36. Ramezani, M., Bathaei, A., and Zahrai, S.M. "Designing fuzzy systems for optimal parameters of TMDs to reduce seismic response of tall buildings", Smart Struct Syst, 20(1), pp. 61-74 (2017).

37. Abdo, M.-B. and Hori, M. "A numerical study of structural damage detection using changes in the rotation of mode shapes", Journal of Sound and vibration, 251(2), pp. 227-239 (2002).

\section{Biographies}

Meysam Ramezani is currently a $\mathrm{PhD}$ candidate at the International Institute of Earthquake Engineering and Seismology, Tehran, Iran. He is an experienced graduate research assistant working in the academic context. His current research interests include structural engineering, vibration control, performance-based design, structural health monitoring, artificial intelligence, and dynamic simulation using OpenSees. He was a strong researcher professional and held MSc degree in Earthquake Engineering from University of Tehran.

Omid Bahar is an Assistant Professor at International Institute of Earthquake Engineering and Seismology (IIEES), Tehran, Iran. He received his $\mathrm{PhD}$ degree in Structural Engineering from Shiraz University, Shiraz, Iran. He spent a short time as a visiting scholar at Professor Kitagawa's Lab. at Hiroshima University during his $\mathrm{PhD}$ research program. He worked on the performance of active structural control during strong ground motions. His main research interests include performance-based design, structural control, and structural health monitoring. 